\title{
Mapping sea surface velocities in the Changjiang coastal zone with advanced synthetic aperture radar
}

\author{
WANG Lihua ${ }^{1}$, ZHOU Yunxuan*, GE Jianzhong ${ }^{1}$, JOHANNESSEN Johnny A. ${ }^{2,3}$, SHEN Fang ${ }^{1}$ \\ ${ }^{1}$ State Key Laboratory of Estuarine and Coastal Research, East China Normal University, Shanghai 200062, \\ China \\ ${ }^{2}$ Nansen Environmental and Remote Sensing Center, Bergen 5006, Norway \\ ${ }^{3}$ Geophysical Institute, University of Bergen, Bergen 5006, Norway
}

Received 24 February 2014; accepted 20 March 2014

(CThe Chinese Society of Oceanography and Springer-Verlag Berlin Heidelberg 2014

\begin{abstract}
Range Doppler velocities derived from the Envisat advanced synthetic aperture radar (ASAR) wide swath images are analyzed and assessed against the numerically simulated surface current fields derived from the finite volume coastal ocean model (FVCOM) for the Changiiang Estuary. Comparisons with the FVCOM simulations show that the European Space Agency (ESA) Envisat ASAR based Doppler shift anomaly retrievals have the capability to capture quantitative information of the surface currents in the Changjiang Estuary. The uncertainty analysis of the ASAR range Doppler velocity estimates are discussed with regard to the azimuthal and range bias corrections, radar incidence angles, inaccuracy in the wind field corrections and the presence of rain cells. The corrected range Doppler velocities for the Changjiang Estuary area are highly valuable as they exhibit quantitative expressions related to the multiscale upper layer dynamics and surface current variability around the East China Sea, including the Changjiang Estuary.
\end{abstract}

Key words: advanced synthetic aperture radar, Changjiang coast, Doppler centroid anomaly, range Doppler velocity, finite volume coastal ocean model

Citation: Wang Lihua, Zhou Yunxuan, Ge Jianzhong, Johannessen Johnny A., Shen Fang. 2014. Mapping sea surface velocities in the Changjiang coastal zone with advanced synthetic aperture radar. Acta Oceanologica Sinica, 33(11): 141-149, doi: 10.1007/ s13131-014-0563-x

\section{Introduction}

It is highly necessary to have a good regular observing system for a high resolution (about $1 \mathrm{~km}$ ) surface current measurements. From an economic, ecological and hydrodynamic point of view, such data are also of utmost importance for assimilation in ocean and shelf circulation models in order to provide adequate predictions of the continuous changes of the estuary. Several technologies are currently used to monitor the sea surface currents, including current meter moorings, drifters, acoustic Doppler current profilers (ADCP) as well as remote sensing methods from satellites and ground based high frequency radar (HF) systems. These instruments and technologies have their inherent strength and limitations as discussed by Pandian et al. (2010). Geostrophic currents on a spatial scale of $25 \mathrm{~km}$ retrieved from a satellite altimetry (Kerbaol and Collard, 2005), for instance, are now being applied routinely in global and regional circulation models. However, in coastal regions the resolution is too course which thus limits the use of this method. High-resolution imaging radars, on the other hand, have demonstrated promising capabilities for the retrieval of surface current estimates on scales from 2 to $10 \mathrm{~km}$ (Chapron et al., 2005; Johannessen et al., 2008; Romeiser and Thompson, 2000; Rouault et al., 2010). Two methods have emerged notably the along-track interferometry that requires a split antenna (Romeiser and Thompson, 2000; Romeiser et al., 2010; Thompson and Jensen,
1993) and the single-antenna SAR Doppler technology (Chapron et al., 2005). In both cases, the basic concept originates from the fact that every detected moving target produces a Doppler frequency shift proportional to its relative velocity towards the radar platform. Chapron et al. (2004) pioneered the single-antenna Envisat ASAR Doppler measurements of moving ocean surfaces, to extract and analyze the line-of-sight radar-detected velocity of the ocean surface roughness (small-scale disturbances such as capillary waves). Furthermore, they showed that the Doppler centroid anomalies recorded by the ASAR are of a geophysical nature. The resulting Doppler anomaly contains information solely related to the motion of the sea surface roughness elements, which reflects the combined action of wind, waves and currents. The corresponding SAR range Doppler surface current measurements have been successfully applied to distinct surface current regimes, e.g. the Agulhas Current (Johannessen et al., 2008; Rouault et al., 2010), the Gulf Stream (Chapron et al., 2005) and the Norwegian Atlantic Current (Hansen et al., 2011). The SAR will therefore have an increasingly important role to play in quantitative studies of ocean surface current features. The development of the SAR Doppler technology, moreover, offers new possibilities to observe and model mesoscale oceanic processes and coastal current phenomena.

The Changjiang Estuary is built out by fluvial deposits, shaped by tides and river runoff. It is a third-order bifurcated

Foundation item: The National Basic Research Program (973 Program) of China under contract No. 2010CB951204; European Space Agency-Ministry of Science and Technology of the People's Republic of China Dragon 3 Cooperation Programme under contract No. 10593; the State Key Laboratory of Estuarine and Coastal Research, East China Normal University of China under contract No. SKLEC-2012KYYW02; the 111 Project under contract No. B08022

*Corresponding author, E-mail: zhouyx@sklec.ecnu.edu.cn 
system, with four outlets to the East China Sea (ECS). The estuary water depth varies from 5.0 to $50.0 \mathrm{~m}$ (Fig. 1). Its tides are typically semidiurnal. Both natural (deposition of fluvial sediment, tides, waves, saline water intrusion, and river runoff) and anthropogenic (reservoir construction, deep water navigation channel and its guide bunds, large-scale water storage basin, land reclamation) factors contribute to the local changes of the coastlines, topography and estuarine morphology, and hence also the hydrodynamics of the estuary and the adjacent coastal area. The Changjiang estuarine and coastal waters constitute a mixed environment with fresh, brackish and salt water, sediments, nutrients, and other substances, and with physical phenomena, such as estuarine circulation, turbidity maxima, mouth bar formation and marine fronts (Li et al., 1994; Wu and $\mathrm{Zhu}$, 2010). A dominant regional driving mechanism for the Changjiang Estuary comprises the movement of the Changjiang Diluted Water (CDW) through the estuary and over the inner shelf of the ECS, as well as related physical factors such as tides, waves and seabed topography. In addition, coastal engineering works (e.g., the deep waterway project, reclamation projects and reservoir building) have significantly changed the local coastlines and topography (Yang et al., 2006), and hence the corresponding hydrodynamics.

Around the Changjiang Estuary and the inner shelf of the ECS, an integrated high-resolution numerical model system based on the finite-volume coastal ocean model (FVCOM) has been implemented with the aim to resolve the multiscale dynamics, including regional, shelf and estuarine circulations (Ge et al., 2012; Ge et al., 2013). The core of this simulation system is a Changjiang Estuary FVCOM hydrodynamic model. It is forced with realistic input data on the outer circulation in the ECS, and a finite-volume dike-groyne treatment algorithm is incorporated to reveal the hydrodynamics around the coastal structures in the river mouths (Ge et al., 2012). This model system has been validated against various field observations, yielding promising results (Ge et al., 2013). The numerically simulated velocities are therefore used to intercompare and validate the sea surface current measurement retrieved from the ASAR WSM data.

In this paper, we briefly present data and an approach to derive geophysical range Doppler velocities. The simulated velocity fields derived from the validated unstructured-grid FVCOM that provides excellent downscaling capabilities to recover the high-resolution dynamics in the Changjiang Estuary are also presented, followed by an intercomparison and assessment of the range Doppler velocity fields. Finally, we give the conclusions and an outlook.

\section{Methods}

The ASAR data acquired in the Changjiang Estuary are from the European Space Agency (ESA) Envisat advanced synthetic aperture radar (ASAR) and involve medium-resolution wide

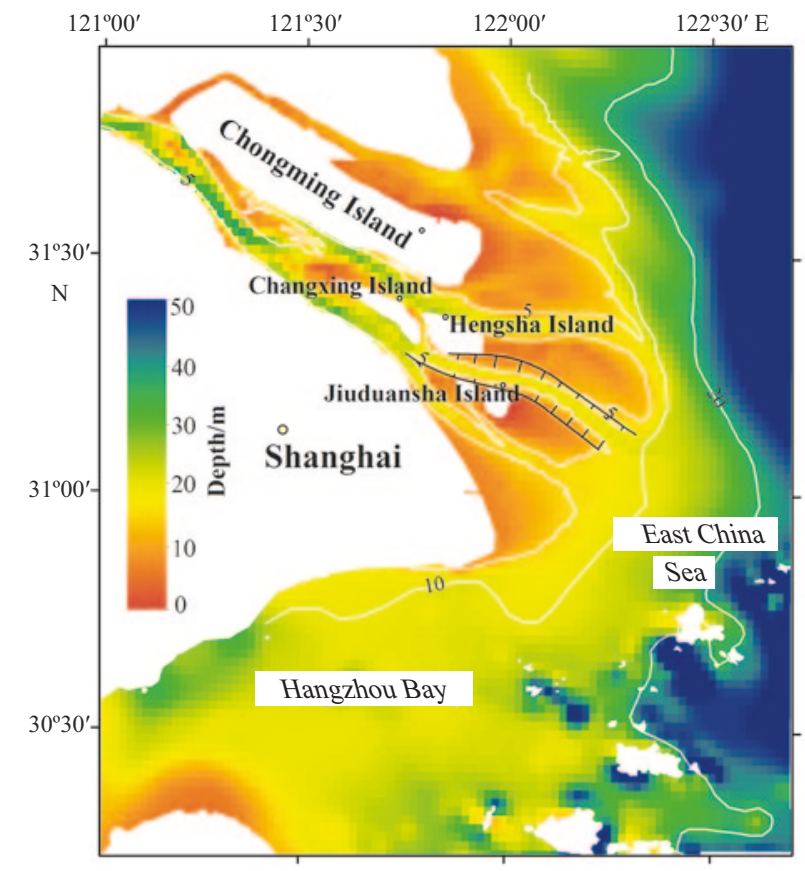

Fig.1. Location of the Changjiang Estuary and its seabed topography.

swath mode (WSM) scenes. The ASAR WSM imagery is systematically generated using the ScanSAR technology and processed to $150 \mathrm{~m}$ resolution with a swath width of approximately $420 \mathrm{~km}$ spanning a range of incidence angles from $16^{\circ}$ to $43^{\circ}$ relative to the surface normal. Over the Changjiang Estuary, one descending (31 January 2005, morning local time) and two ascending (5 February 2005 and 9 May 2006, both evening local time) ASAR WSM images have been obtained to derive the surface range Doppler velocity fields. In addition, two ASAR images (one descending 3 February 2005 and one ascending 12 May 2006), have been acquired from adjacent orbits/acquisition time with sufficient land cover. Both are used as reference data to remove the Doppler frequency biases from the other three scenes (Table 1).

The Doppler centroid frequency of the SAR signal $f_{\mathrm{Dc}}$ is a key input parameter to the processing of ASAR data. For the ASAR WSM data, the fine Doppler centroid, or the fractional pulse repetition frequency (PRF) part of the Doppler centroid, is determined by Madsen's algorithm (Madsen, 1989), since it is simple to implement and computationally efficient. Then a refinement of the fractional Doppler estimation is derived using Look Power Balancing technologies (Michael, 1996). The PRF diversity method is applied to different beams in order to

Table 1. Envisat ASAR WSM products description

\begin{tabular}{ccccccc}
\hline Acquisition time & Polarization & $\begin{array}{c}\text { Doppler pixel numbers } \\
\text { (range by azimuth) }\end{array}$ & Orbit & Track & Pass \\
\hline 2005-01-31 01:53:21 & VV & $100 \times 52$ & 15273 & 189 & descending & main scene \\
2005-02-03 01:59:02 & VV & $100 \times 51$ & 15316 & 232 & descending & reference scene \\
2005-02-05 13:47:28 & VV & $100 \times 52$ & 15352 & 268 & ascending & main scene \\
2006-05-09 13:53:06 & VV & $100 \times 52$ & 21908 & 311 & ascending \\
2006-05-12 13:58:50 & VV & $100 \times 52$ & 21951 & 354 & main scene \\
\hline
\end{tabular}


resolve the Doppler ambiguity (Chang and Curlander, 1992). A systematic grid of Doppler centroid frequencies contains 100 pixels in a range direction and a given number in an azimuth direction depending on the scene coverage. The cross-track pixel spacing in the range direction is about $9 \mathrm{~km}$ in near range and $3.5 \mathrm{~km}$ in far range, while in the azimuth direction the spacing is about $8 \mathrm{~km}$.

The satellite along-track velocity relative to the rotating earth induces a frequency shift $f_{\mathrm{Dp}}$. The Doppler orbitography and radio-positioning integrated by satellite instrument (DORIS) is a microwave tracking system that can be utilized to determine the precise location of the Envisat satellite. The Envisat restituted attitude file (AUX_FRA_AX) is handled automatically by the orbit propagation CFI software (Envisat CFI Software). This file contains the AOCS parameters and information about the attitude of the spacecraft (roll, pitch and yaw). Therefore, the $f_{\mathrm{Dp}}$ and footprint geolocation parameters can be accurately calculated for any look angle and any orbit time using the CFI software.

The estimation error $f_{\text {err }}$, such as antenna mispointing, radial discontinuities, areas of low signal-to-noise ratio (SNR), strong discrete targets and Doppler estimator bias, that contaminates the geophysical Doppler shift information, must be eliminated first (Chapron et al., 2005; Dagestad et al., 2010; Hansen et al., 2011; Johannessen et al., 2008; Mouche et al., 2012). It is worth mentioning that the spatial corresponding relationship between Doppler grid and backscatter scene can be determined by the geolocation grid annotation data set (ADS) in the metadata of ASAR products (Fig. 2). Moreover, the Doppler block size in the azimuth direction is defined from first zero Doppler time to last zero Doppler time, in the range direction it is 356 divided by the rate of range sampling and this rate is given in the main processing parameter ADS in the metadata of the ASAR. Considering that the average elevation of Shanghai in the Changjiang coastal delta is only about $4 \mathrm{~m}$ above the sea level, the error in the Doppler frequency introduced by this topography will be negligible. Therefore, as long as there are enough land pixels for each range line number of the scene, the land will always be a good reference source to remove the biases due to the deviation of actual radar beam pointing angle and the theoretical pointing angle (Hansen et al., 2011). The scenes from the adjacent orbits/acquisition time are therefore selected as reference data to remove the Doppler anomaly biases depending on an elevation angle. The ASAR WSM image on February 32005 with sufficient land pixels is applied to remove the Doppler biases of the ASAR WSM scenes on 31 January and 5 February 2005; while the ASAR on May 122006 is used to remove the biases of the ASAR on 9 May 2006. The details of the Doppler centroid anomaly are given by Hansen et al. (2011).

Wind-induced streaks are manifested in the ASAR scenes used in this study. Consequently, the wind directions can be directly derived from the ASAR images using 2-D fast Fourier transformation (FFT) in the spectral domain (Gerling, 1986; Horstmann et al., 2004; Lehner et al., 2000; Vachon and Dobson, 1996). Hence, with the wind direction derived from the ASAR data, the wind speed can be reliably retrieved using a C-band model (CMOD4) together with the known geometry parameters (e.g., the incidence angle and the azimuth angle with respect to the wind direction). In turn, the ASAR derived-wind vectors employed in combination with the C-band Doppler shift (CDOP) function (Mouche et al., 2012) yields an estimation of the wind

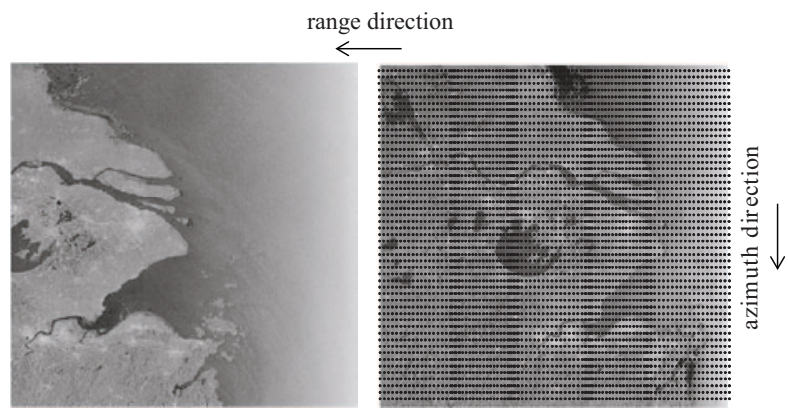

Fig.2. The NRCS of the ASAR WSM scene over Changiiang coast on 31 January 2005 (left) and 3 February 2005 (right). The latter has the Doppler centroid grid (points) superimposed. Arrows mark the azimuth and range directions.

contribution to the retrieved range Doppler velocities. This wind-induced contribution $f_{\mathrm{w}}$ is then removed from the Doppler centroid anomaly.

The geophysical Doppler anomaly $f_{\mathrm{g}}$ can be obtained with the following Eq. (1) and converted with Eq. (2) to the surface current fields:

$$
\begin{aligned}
& f_{\mathrm{g}}=f_{\mathrm{Dc}}-f_{\mathrm{Dp}}-f_{\mathrm{err}}-f_{\mathrm{w}}, \\
& V=-\varpi f_{\mathrm{g}} / k_{\mathrm{E}} \sin \theta,
\end{aligned}
$$

where $k_{\mathrm{E}}$ is $112 \mathrm{~m}^{-1}$ for the radar wavelength of $5.6 \mathrm{~cm}$ of the Envisat ASAR instrument; and $\theta$ denotes the incidence angle.

\section{Results}

Figures 3, 4 and 5 show $f_{\mathrm{Dc}}, f_{\mathrm{Dp}}$, the raw Doppler anomaly $\left(f_{\mathrm{Dc}}-f_{\mathrm{Dp}}\right)$ and the geophysical Doppler centroid anomaly $f_{\mathrm{g}}$ from ASAR WSM image acquired over the Changjiang Estuary on 31 January 2005, 5 February 2005 and 9 May 2006, respectively. The large variability of the raw Doppler centroid anomaly is observed (Figs 3c, $4 c$ and 5c), even over land where the Doppler centroid anomaly should be 0 . The strong normalized radar cross-section (NRCS) gradient along the azimuth direction is one of the main sources of bias in the Doppler estimation and is particularly pronounced along the coastline in all three cases considered. Moreover, the erroneous Doppler frequencies aligned in the azimuth direction are also evident as vertical stripes of enhanced/reduced Doppler anomalies at the transition region between different subswaths. Apart from this, additional artifacts can also contribute to the biases. After the error correction in the azimuth and range directions, the root mean square (RMS) offset over land is reduced from 24.5, 21.4 and $25.5 \mathrm{~Hz}$ of the raw Doppler anomaly to 10.8, 9.3 and $12.2 \mathrm{~Hz}$ (Table 2). Yet, the Doppler anomaly may obtain incorrect values due to strong azimuthal NRCS gradients, influence of rain cells, and region close to land (Chapron et al., 2005; Hansen et al., 2011; Rouault et al., 2010), with a negative impact on the overall performance of the ASAR-derived surface current velocities. Removing these outlying values, the RMS offset over land is further reduced to 6.2, 6.1, and $5.3 \mathrm{~Hz}$, respectively (Table 2).The geophysical Doppler anomaly (Figs $3 \mathrm{~d}$ and $4 \mathrm{~d}$ ) after the removal of the wind-induced signal is converted with Eq. (2) to the sur- 

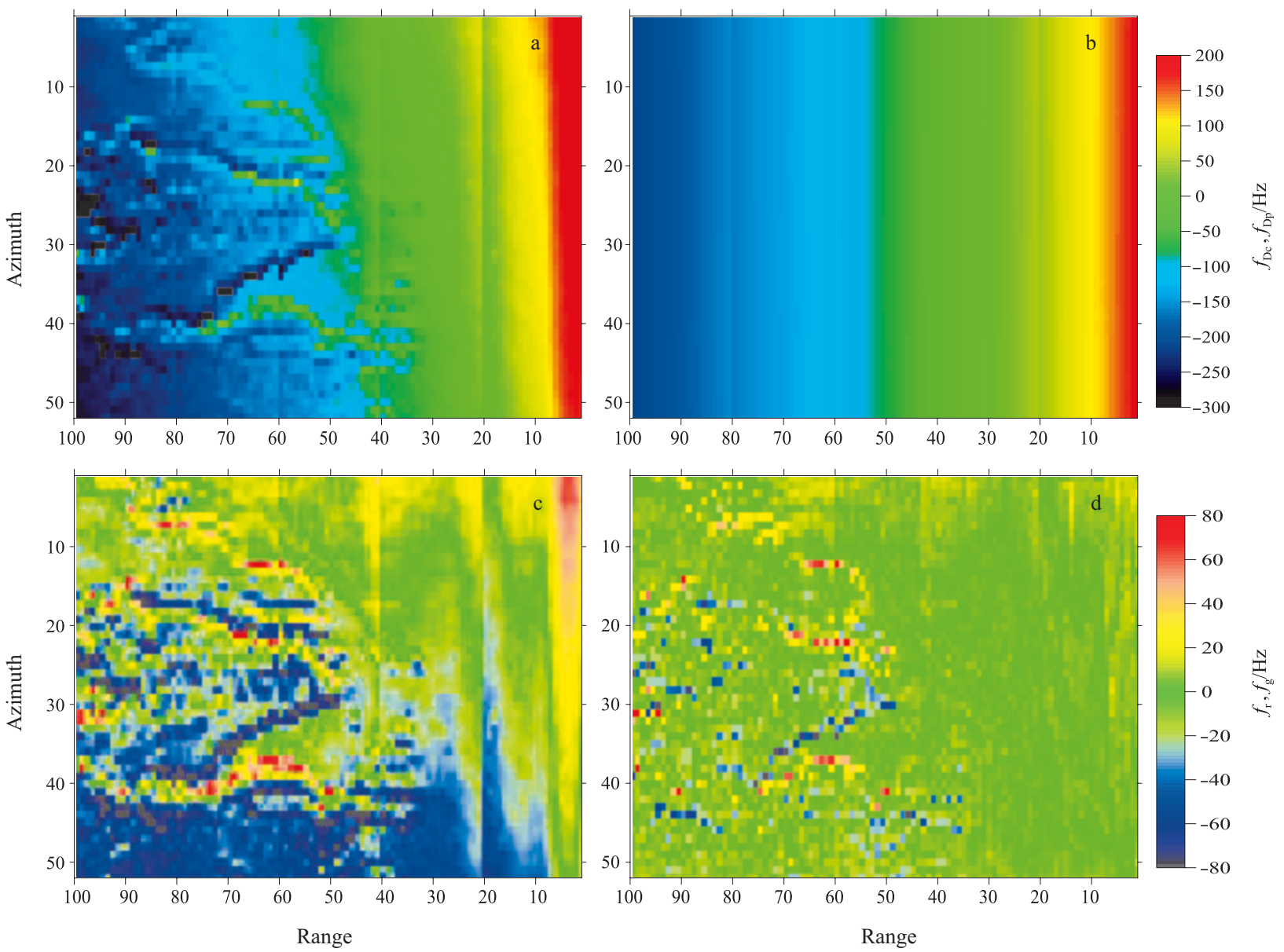

Fig.3. The Doppler centroid grid from an Envisat ASAR WSM scene acquired on 31 January 2005. a. $f_{\mathrm{Dc}}$, b. $f_{\mathrm{Dp}}$, c. the raw Doppler centroid anomaly $f_{\mathrm{r}}$ and d. the geophysical Doppler centroid anomaly $f_{\mathrm{g}}$.

face current fields shown in Figs 6a and b, respectively.

In addition, a nesting of the local computational domain $\left(28.3^{\circ}\right.$ to $34.3^{\circ} \mathrm{N}$ and $120.0^{\circ}$ to $124.5^{\circ} \mathrm{E}$ ), including the Changjiang Estuary within the FVCOM framework, gives a mesh of about 100000 cells with a finest resolution of about $200 \mathrm{~m}$ located around the deep waterway structures. The surface current at the time of the ASAR WSM scene acquisition is calculated by interpolation over the $1 \mathrm{~h}$ resolution output of the model and superimposed on the results of the sea surface current retrieved from the ASAR WSM data (Figs 7a and $b$ ).

\section{Discussion}

In this study, the Doppler method yields estimates with a resolution (azimuth, range) of about $8 \mathrm{~km} \times 4 \mathrm{~km}$. We adopt the convention that the positive range directed velocity values corresponds to the sea surface velocities away from the radar, whereas negative values indicate a flow towards the radar. There are a number of error sources that influence the accuracy of the Doppler velocities when retrieving sea surface Doppler velocities from ASAR images. Most of these errors are caused by the strong azimuthal NRCS gradients, the low radar incidence angle, the inaccuracy in wind field and the presence of rain cells. Note that the Doppler velocity estimates of the scene on 9 May 2006 are not included in the quantitative comparison, because of the apparent strong rain effects that limit the Doppler shift retrieval accuracy (Rouault et al., 2010).

\subsection{ASAR range Doppler velocities}

Figure 6a shows the Doppler velocities derived from the ASAR WSM image on 31 January 2005. There is a distinct shift in a direction located at about $31.5^{\circ} \mathrm{N}$. A southeasterly flow

Table 2. Doppler anomaly RMS bias over land of the scenes

\begin{tabular}{ccccc}
\hline Acquisition time & \multicolumn{3}{c}{ RMS of Doppler anomaly/Hz } \\
\cline { 2 - 5 } of ASAR scene & raw & after azimuthal correction & after bias correction & after outliers removal \\
\hline 31 Jan. 2005 & 24.5 & 19.0 & 10.8 & 6.2 \\
5 Feb. 2005 & 21.4 & 16.1 & 9.3 & 6.1 \\
9 May 2006 & 25.5 & 21.0 & 12.2 & 5.3 \\
\hline
\end{tabular}



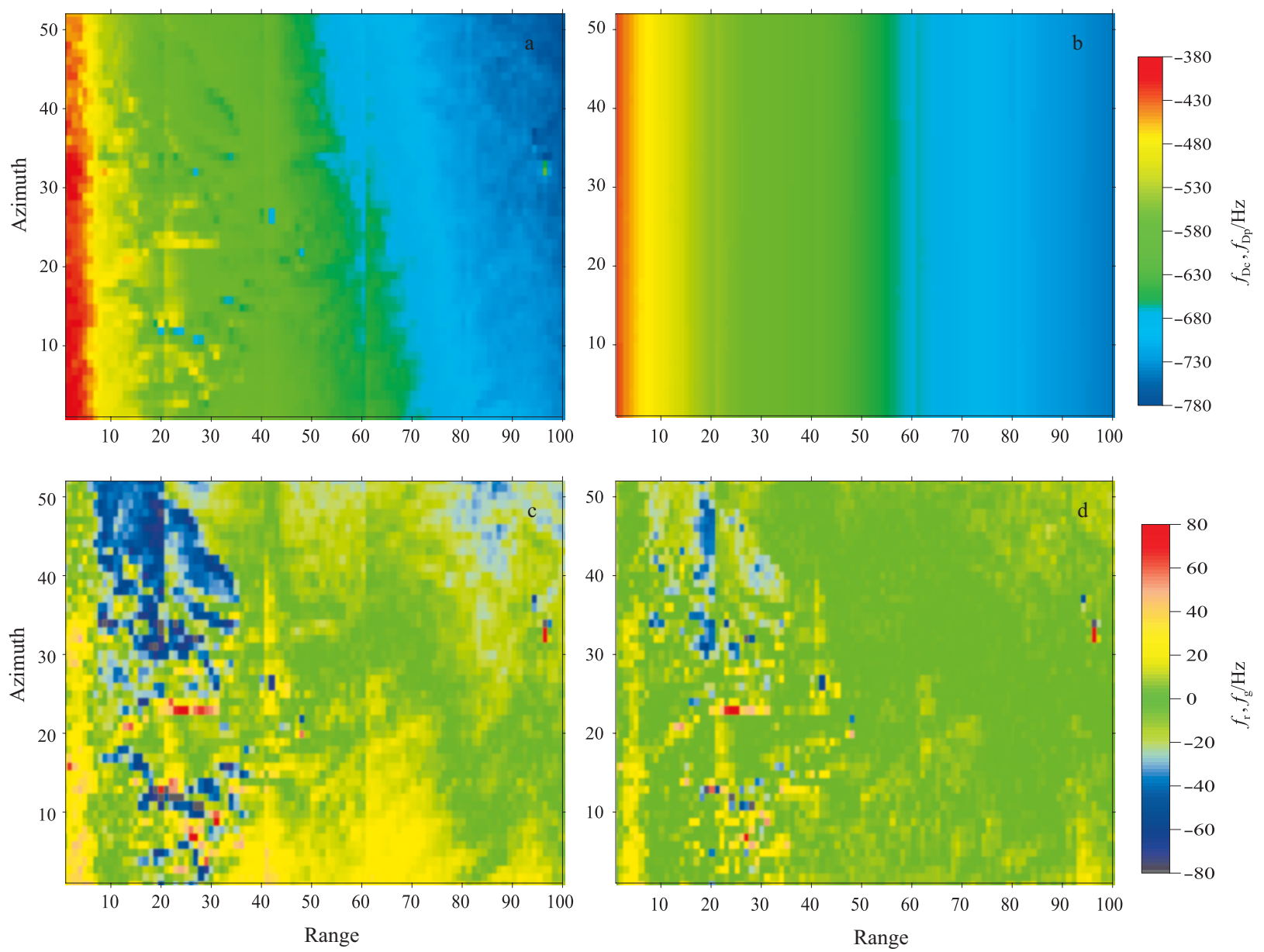

Fig.4. The Doppler centroid grid from an Envisat ASAR WSM scene acquired on 5 February 2005. a. $f_{\mathrm{Dc}}$, b. $f_{\mathrm{Dp}}$, c. the raw Doppler centroid anomaly $f_{\mathrm{r}}$ and d. the geophysical Doppler centroid anomaly $f_{\mathrm{g}}$.

is also encountered in the Hangzhou Bay area. At the time of image acquisition, the wind direction was northwest and the wind speed increased with the distance from the shore, from 8 to $11 \mathrm{~m} / \mathrm{s}$. This wind field exhibits a qualitative correlation with the SAR backscatter signal (not shown). This is particularly the case at about $2 \mathrm{~h}$ after a low tide at Sheshan, Niupijiao, and Dajishan Stations, when the tidal currents begin to increase. At the Tanhushan Station it happens exactly at the low tide, when the tidal currents should be low; yet the Doppler velocity is relatively strong according to the ASAR velocity map, which is probably caused by the underwater terrain and the combined action of the wind, the waves and the currents.

Figure $6 \mathrm{~b}$ shows the range Doppler velocity from the image acquired on 5 February 2005. The ASAR velocities at the scene acquisition time are mostly negative in the sector from $122.5^{\circ} \mathrm{E}$ to west, with a magnitude ranging from 0.2 to $1.2 \mathrm{~m} / \mathrm{s}$. This corresponds to a sea surface current moving to the west/ southwest. In the sector $122.5^{\circ} \mathrm{E}$ to east, the ASAR velocities are mainly positive, indicating a surface current towards east/ northeast. At the scene acquisition time, the wind directions were from the northeast, i.e., towards the antenna. The wind speeds ranged from 9 to $11 \mathrm{~m} / \mathrm{s}$. The areas of the strong negative Doppler velocity appear in the Hangzhou Bay. The ASAR velocity is $-0.25 \mathrm{~m} / \mathrm{s}$ at the Tanhushan Station, $40 \mathrm{~min}$ after a high tide. At the remaining four tidal stations, the values are quite variable even though they all occurred at some 2-3 $\mathrm{h}$ after the high tide. Therefore any Doppler velocity map such as Fig. $6 \mathrm{~b}$ represents wind and current patterns in a rather complicated way. Local changes in the wind field complicate the geophysical interpretation of the Doppler velocity (Chapron et al., 2005).

The observed velocity fields in two cases demonstrate that the strongest is found in the Hangzhou Bay. Velocities there, amounting to $0.8-1.0 \mathrm{~m} / \mathrm{s}$, are mainly affected by the interaction between the Changjiang runoff and tide. As all three cases concern spring tide, the observed velocities represent the most intense flow. The capability of the ASAR to capture strong surface currents was also demonstrated in the Agulhas return current region (Rouault et al., 2010). Large ASAR velocities, however, are always associated with strong gradients in the backscatter signal. If the bias correction of the Doppler shift anomaly in the azimuth direction is not sufficient, it negatively affects the range Doppler velocity estimation. Especially landsea boundaries this needs more attention and analysis.

The ASAR imaging geometry with respect to the velocity field is highly important to the quality of the Doppler retriev- 

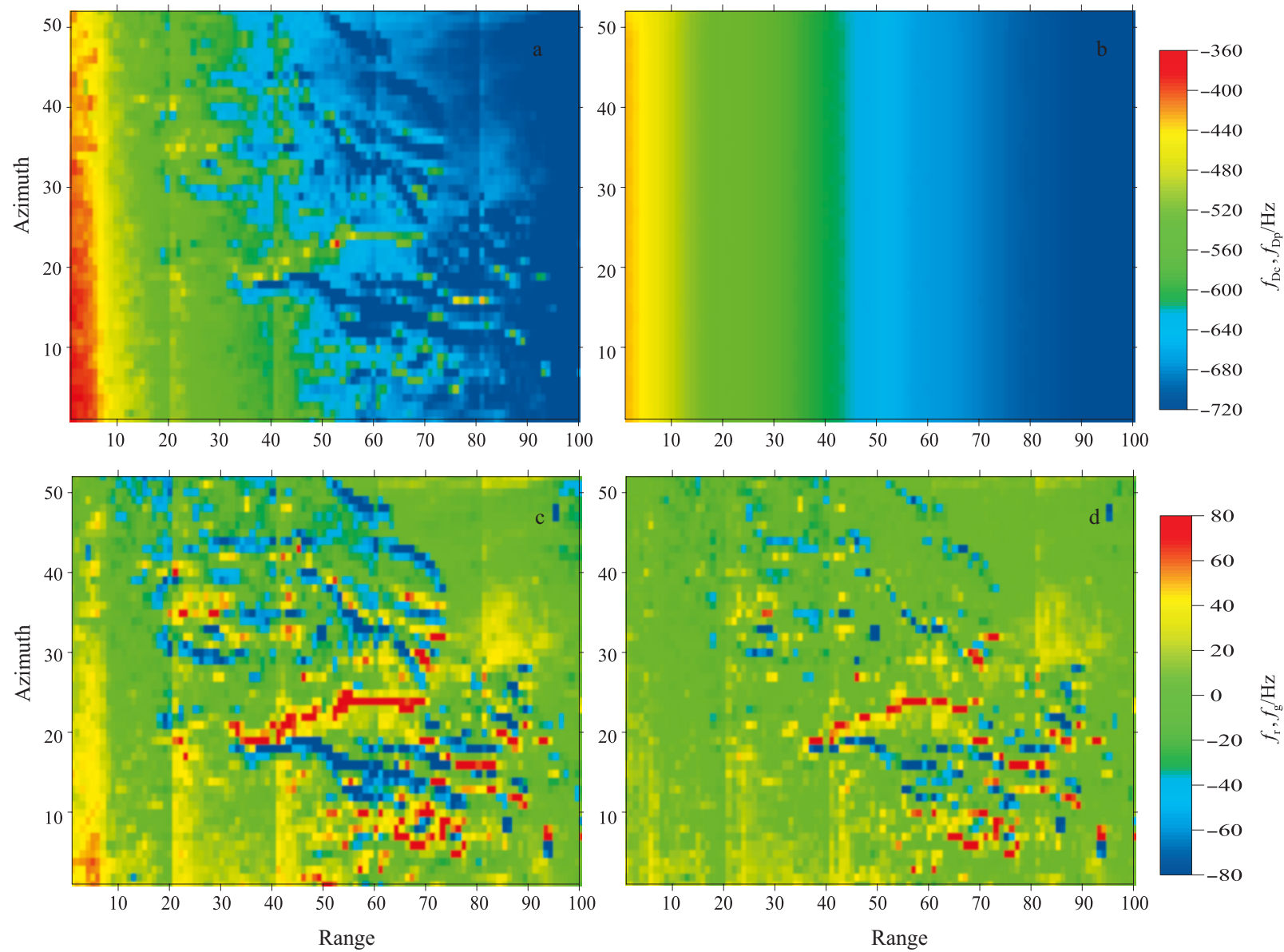

Fig.5. The Doppler centroid grid from an Envisat ASAR WSM scene acquired on 9 May 2006. a. $f_{\mathrm{Dc}}$, b. $f_{\mathrm{Dp}}$, c. the raw Doppler centroid anomaly $f_{\mathrm{r}}$ and d. the geophysical Doppler centroid anomaly $f_{\mathrm{g}}$.
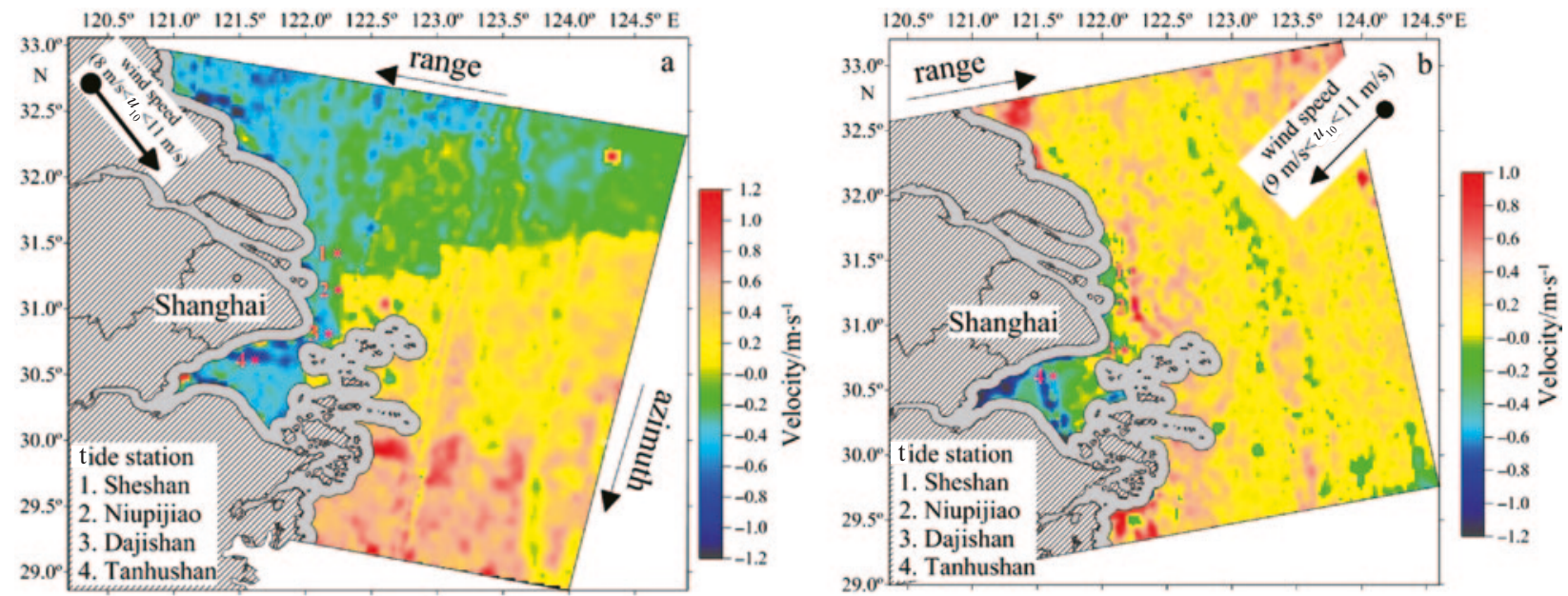

Fig.6. Surface Doppler velocity from the ASAR WSM scene on 31 January 2005 (a) and on 5 February 2005 (b).

als. The descending track configuration is well suited to capture the spatial variations of the current field in the Changjiang Estuary, since it measures the surface flow along a southeast/ northwest axis and Changjiang Estuary mouth is imaged at the high incidence angle of the ASAR (Fig. 6a). 

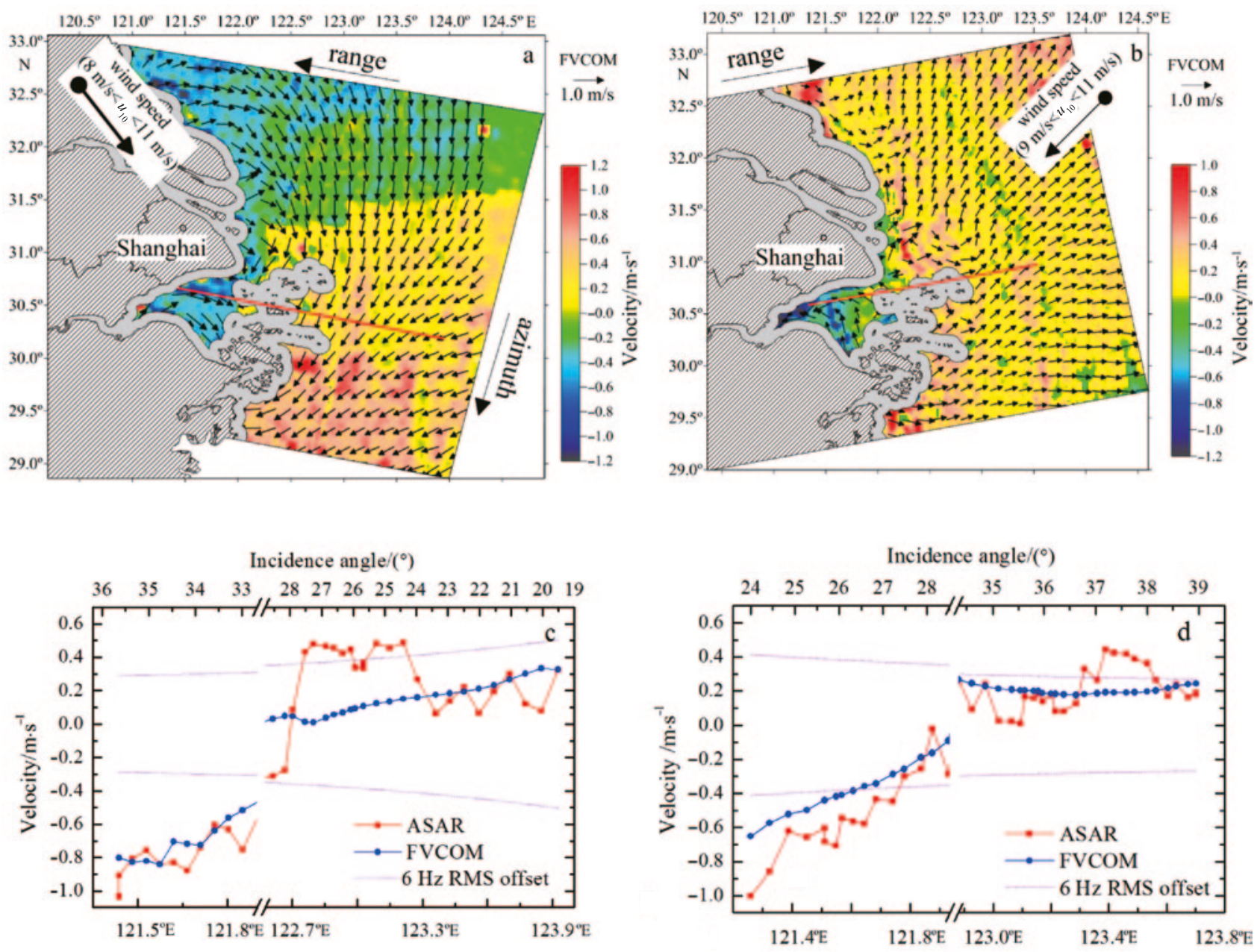

Fig.7. Surface Doppler velocity from the ASAR WSM scene on 31 January 2005 (a) and on 5 February 2005 (b) with the corresponding simulated surface currents based on FVCOM superimposed as arrows (strength indicated by their lengths). Transects of ASAR range velocity, FVCOM velocity on 31 January 2005 (c) and on 5 February 2005 (d).

\subsection{Uncertainty analysis of ASAR Doppler velocities}

When the strong Doppler variations go across the antenna lobe, a NRCS gradient will bias the $f_{\mathrm{Dc}}$ to the Doppler emerging from the brighter part of the illuminated ground. This effect is most prominent in cases with large variations in the radar backscatter within the azimuth beamwidth of the SAR antenna (e.g., when the SAR is imaging a land-water boundary). Consequently, the estimated Doppler centroid could be distorted due to the relatively strong or weak intensity away from the center of the radar beam (Chapron et al., 2005; Li et al., 1985). The residual strong velocity in the first Doppler pixel from the coast still contains an artifact from the imperfect correction of the azimuth bias from the NRCS gradient even if the azimuth bias has been corrected and mentioned before, so we removed these with an $10 \mathrm{~km}$ extended land mask in Figs $6 \mathrm{a}$ and $\mathrm{b}$.

The RMS offset of these scenes after an error correction was found to be about $6.0 \mathrm{~Hz}$, which corresponds to a horizontal Doppler velocity of about $24 \mathrm{~cm} / \mathrm{s}$ at $40^{\circ}$ incidence angle (in far range) and $48 \mathrm{~cm} / \mathrm{s}$ at $20^{\circ}$ incidence angle (in near range). In order to retrieve an accuracy of a surface velocity of less than $30 \mathrm{~cm} / \mathrm{s}$, the use of the incidence angle above $34^{\circ}$ is required.
This is demonstrated in Figs 7c and d. At low radar incidence angles, the backscatter signatures are stronger and dominated by larger and faster roughness elements (Collard et al., 2008). Consequently, the contribution of vertical motions to the velocity increases at low incidence angles, which make the ASAR horizontal velocity retrieval from the Doppler centroid anomaly more challenging (Fig. 6b).

The sea surface wind fields obtained from blending of the National Center for Environmental Prediction (NCEP) and the European Centre for Medium-range Weather Forecasts (ECMWF) have a spatial resolution on a global $0.25^{\circ}$ grid and a time resolution of $6 \mathrm{~h}$. We first interpolated these blended sea surface winds to the ASAR-derived spatial wind fields and acquisition time using the natural neighbor method. Comparisons between these blended sea surface winds and ASAR-retrieved wind vectors have shown an RMS error of about $8^{\circ}$ in the wind direction and a wind speed difference of about $1 \mathrm{~m} / \mathrm{s}$. According to the CDOP, each of these wind-induced errors would result in a maximum error in an ASAR range velocity of about $0.1 \mathrm{~m} / \mathrm{s}$. Moreover, the ASAR surface current velocities are particularly sensitive to the inaccuracies in the wind direction; even small errors 
in the wind direction can significantly reduce the accuracy of the ASAR surface current velocity (Chapron et al., 2005; Collard et al., 2008; Johannessen et al., 2008; Rouault et al., 2010). Thus, the wind vectors directly retrieved from the ASAR images, in turn, provide more consistent estimate of the ocean surface currents, mostly related to a more consistent wind speed, the wind direction being mostly driven by the first guess external atmospheric model.

\subsection{Comparisons between ASAR and FVCOM velocities}

Considering there is no simultaneous in situ observation data available, the comparisons between ASAR (both ascending and descending pass) and FVCOM velocities are provided to examine and assess the capability of the ASAR-based Doppler centroid anomaly measurements for the retrieval of the surface currents. The inspection of the FVCOM results exhibits the same general surface velocity field both in magnitude and direction (see Figs $7 \mathrm{a}$ and $\mathrm{b}$ ). Two transects of the ASAR and FVCOM surface velocity maps (Figs $7 \mathrm{a}$ and $\mathrm{b}$ ) have been extracted for a quantitative comparison, one descending scene transect at about $30.5^{\circ} \mathrm{N}$ on 31 January 2005 , and one ascending image at about $30.7^{\circ} \mathrm{N}$ on 5 February 2005.The positions of transects are indicated as red lines in Figs $7 \mathrm{a}$ and b, respectively. Based on a simple geometrical consideration of ASAR viewing angles in ascending and descending satellite passes, the FVCOM velocities are firstly rotated into the ASAR look direction and flight direction, using the following two equations:

$$
\begin{aligned}
& u_{\mathrm{l}}=u \cos \alpha+\nu \sin \alpha, \\
& v_{\mathrm{f}}=-u \sin \alpha+v \cos \alpha,
\end{aligned}
$$

where $u_{1}$ is the FVCOM velocity component in the ASAR look direction; $v_{\mathrm{f}}$ the component in the azimuth direction; $u$ and $v$ are the meridional and zonal current components of the FVCOM velocity, respectively; and $\alpha$ is the angle rotating anticlockwise from geographical east to the radar look axis.

The ASAR-derived surface current directions in these two transects are in good agreement with the FVCOM-results on 31 January and 5 February 2005 (Figs 7c and d) both regarding location of southeasterly and northwesterly flow directions. Overall, the comparison of the strength of the surface current is also reasonable good provided the speed is above $\pm 0.2 \mathrm{~m} / \mathrm{s}$. The maximum surface current velocities derived from the ASAR in both cases reach about $1.0 \mathrm{~m} / \mathrm{s}$, whereas the FVCOM velocities are only $0.8 \mathrm{~m} / \mathrm{s}$ on 31 January 2005 and $0.6 \mathrm{~m} / \mathrm{s}$ on 5 February 2005. The maximum difference is $0.42 \mathrm{~m} / \mathrm{s}$ at about $122.7^{\circ} \mathrm{E}$ on 31 January 2005, and $0.35 \mathrm{~m} / \mathrm{s}$ at $121.2^{\circ} \mathrm{E}$ on 5 February 2005 . The corresponding incidence angle is $27.5^{\circ}$ and $24.0^{\circ}$, respectively; both are below a radar incidence angle of $30^{\circ}$. In this regard, we further calculate the average magnitude discrepancy between the ASAR Doppler velocities and the FVCOM results below and above $30^{\circ}$ radar incidence angle. It is shown that the difference is $0.09 \mathrm{~m} / \mathrm{s}$ on 31 January 2005 and $0.10 \mathrm{~m} / \mathrm{s}$ on 5 February 2005 above incidence angle $30^{\circ}$; however it can increase to $0.24 \mathrm{~m} / \mathrm{s}$ on 31 January 2005 and $0.18 \mathrm{~m} / \mathrm{s}$ on 5 February 2005 below incidence angle $30^{\circ}$. This result corroborates the discussion outlined in the section of uncertainty analysis of the ASAR Doppler velocity and is also consistent with the previous findings (Chapron et al.,2005; Collard et al., 2008; Johannessen et al., 2008) revealing a sudden increase in the ASAR Doppler velocity error below a radar incidence angle of $30^{\circ}$.
In the FVCOM velocity field of 31 January 2005 (Fig. 7a), the dominant flow direction is to the south-southwest in the sector eastward from $122.2^{\circ} \mathrm{E}$. In comparison the ASAR observations only sense the surface current velocity in the range plain, towards west-northwest or east-southeast. Consequently, the ASAR range velocity is rather weak $(< \pm 0.15 \mathrm{~m} / \mathrm{s})$ in the region north of about $31.0^{\circ} \mathrm{N}$. In contrast the FVCOM velocity map of 5 February 2005 (Fig. 7b), mainly reveals motions towards east and northeast eastward from $122.5^{\circ} \mathrm{E}$. This coincides well with the line-of-sight direction of the ASAR, at least south of about $31.5^{\circ} \mathrm{N}$. Hence the extraction of the surface range Doppler velocity is more reliable and provides a better estimate of the real local sea surface current.

Although the Doppler velocity estimates contain spatial variations, a clear correlation exists between the local FVCOM current and the ASAR Doppler velocity at the Changjiang Estuary reaching 0.56 for the case of 31 January 2005 and 0.59 for the case of 5 February 2005. In comparison, Hansen et al., (2011) reported a correlation coefficient between the ASAR-derived velocities and the recording current meter (RCM) as well as the AVISO range velocities for the Norwegian Atlantic slope current of about 0.62 and 0.56 . In consistence with previous findings (Hansen et al., 2011; Johannessen et al., 2008; Rouault et al., 2010), the accuracy of these measured Doppler velocity fields are influenced by the radar wavelength, the incidence angle, the polarization and the antenna footprint size. Nevertheless, the range Doppler-based surface velocity retrieval method can definitely be of help to retrieve mesoscale ocean dynamics and effectively reveal sea surface signatures associated with changes in the local environmental conditions.

\section{Conclusions}

The geophysical Doppler anomaly from the Envisat ASAR WSM scenes can be obtained using the Doppler centroid grid for the ASAR processing, since the attitude of the ASAR platform is well known (Chapron et al., 2005). Yet, biases are contaminating the Doppler centroid shift that, in turn, affects the retrieval of the range Doppler velocity. Careful corrections and bias removal are therefore highly needed to retrieve the reliable estimates of the range Doppler surface velocity and subsequently the surface current. The azimuth bias removal from the NRCS gradients and the range bias removal from the reference data have been presented for three different ASAR scenes at the Changjiang Estuary. The RMS offset of the corrected Doppler anomaly is approximately to $6.0 \mathrm{~Hz}$, corresponding to a horizontal Doppler velocity of $29 \mathrm{~cm} / \mathrm{s}$ at the $35^{\circ}$ incidence angle.

The orientation of the Envisat ASAR tracks with respect to the Changjiang River outflow, together with the Changjiang Estuary imaged at the high incidence angle implies that the descending Envisat tracks are the most attractive for the range Doppler shift method. Moreover, the ASAR surface current velocities are particularly sensitive to inaccuracies in the wind correction. Using wind fields accurately retrieved from the ASAR images yields the most accurate retrieval of the ASAR surface current.

The intercomparison and validation of the ASAR-derived Doppler velocities against the surface velocity field derived from numerical ocean model simulations show promising results. The Doppler measurements therefore have the capability to derive the innovative estimates of the surface velocities at Changjiang Estuary. These Doppler-based velocity retrievals from the ASAR images of the Changjiang Estuary area are valu- 
able as they will contribute to revealing the multiscale dynamics around the East China Sea. Further processes and analyses of the Envisat ASAR data since 2005 have been helpful to examine and undertake routine monitoring of multiscale sea surface dynamics together with the in-situ measurement at the Changjiang Estuary. Furthermore, the ASAR Doppler velocities have the capability to provide sufficiently accurate spatial information for the validation of high resolution coastal models.

A new C-band SAR mission is planned for launch with the ESA Sentinel-1 satellite in May 2014. The Doppler grid will be an official product with a higher accuracy from this mission. Therefore, the potential to bring SAR single-antenna Doppler shift from research applications to an operational tool is likely to materialize in the near future.

\section{Acknowledgements}

This study was supported by the European Space Agency (ESA) ESRIN via the international research fellowship from January to July 2013. The authors would like to thank YvesLouis Desnos, Andy Zmuda and Fabrice Collard for valuable comments and helpful discussions. Also we would particularly thank Huib de Vriend for valuable comments and linguistic checking.

\section{References}

Chang C Y, Curlander J C. 1992. Application of the multiple PRF technique to resolve Doppler centroid estimation ambiguity for spaceborne SAR. IEEE Transactions on Geoscience and Remote Sensing, 30(5): 941-949

Chapron B, Collard F, Ardhuin F. 2005. Direct measurements of ocean surface velocity from space: interpretation and validation. Journal of Geophysical Research, 110: C07008

Chapron B, Collard F, Kerbaol V. 2004. Satellite synthetic aperture radar sea surface Doppler measurements. Proceedings of the 2nd Workshop Coastal and Marine Applications Synthetic Aperture Radar (SAR). Svalbard, Norway: ESA Publications Division

Collard F, Mouche A, Chapron B, et al. 2008. Routine high resolution observation of selected major surface currents from space. Proceedings of the Conference of SEASAR. Frascati, Italy: ESA Publications Division

Dagestad K F, Hansen M W, Johannessen J A, et al. 2010. Inverting Consistent Surface Current Fields from SAR. Frascati, Roma: European Space Research Institute

Envisat CFI Software. http://eop-cfi.esa.int/index.php/mission-cfisoftware/envcfi-software

Ge Jianzhong, Chen Changsheng, Qi Jianhua, et al. 2012. A dike-groyne algorithm in a terrain-following coordinate ocean model (FVCOM): development, validation and application. Ocean Modelling, 47(C): 26-40

Ge Jianzhong, Ding Pingxing, Chen Changsheng, et al. 2013. An integrated East China Sea-Changjiang Estuary model system with aim at resolving multi-scale regional-shelf-estuarine dynamics. Ocean Dynamics, 63(8): 881-900

Gerling T W. 1986. Structure of the surface wind field from the Seasat SAR. Journal of Geophysical Research, 91(C2): 2308-2320

Graber H C, Thompson D R, Carande R E. 1996. Ocean surface features and currents measured with synthetic aperture radar interferometry and HF radar. Journal of Geophysical Research, 101(C11): 25813-25832

Hansen MW, Collard F, Dagestad K F, et al. 2011. Retrieval of sea surface range velocities from Envisat ASAR Doppler centroid measurements. IEEE Transactions on Geoscience and Remote Sensing 49(10): 3582-3592

Hansen M W, Johannessen J A, Dagestad K F, et al. 2011. Monitoring the surface inflow of Atlantic water to the Norwegian Sea using Envisat ASAR. Journal of Geophysical Research, 116: C12008

Horstmann J, Koch W, Lehner S. 2004. Ocean wind field retrieved from the advanced synthetic aperture radar aboard ENVISAT. Ocean Dynamics, 54(6): 570-576

Johannessen J A, Chapron B, Collard F, et al. 2008. Direct ocean surface velocity measurements from space: improved quantitative interpretation of Envisat ASAR observations. Geophysical Research Letter, 35: L22608

Kerbaol V, Collard F. 2005. SAR-derived coastal and marine applications: from research to operational products. IEEE Journal of Oceanic Engineering, 30(3): 472-486

Li F K, Held D N, Curlander J C, et al. 1985. Doppler parameter estimation for spaceborne synthetic-aperture radars. IEEE Transactions on Geoscience and Remote Sensing, 23(1): 47-56

Li Jiufa, Shi Weirong, Shen Huanting. 1994. Sediment properties and transportation in the turbidity maximum in Changjiang estuary. Geographical Research (in Chinese), 13(1): 51-59

Lehner S, Schulz-Stellenfleth J, Schattler B, et al. 2000. Wind and wave measurements using complex ERS-2 SAR wave mode data. IEEE Transactions on Geoscience and Remote Sensing, 38(5): 22462257

Madsen S N. 1989. Estimating the Doppler centroid of SAR data. IEEE Transaction on Aerospace and Electornic Systems, 25(2): 134140

Michael Y J. 1996. Optimal range and Doppler centroid estimation for a ScanSAR system. IEEE Transactions on Geoscience and Remote Sensing, 34(2): 479-488

Mouche A, Dagestad K F, Collard F, et al. 2012. On the use of Doppler shift for sea surface wind retrieval from SAR. IEEE Transactions on Geoscience and Remote Sensing, 50(7): 2901-2909

National Climatic Data Center. http://www.ncdc.noaa.gov/oa/rsad/ air-sea/seawinds.html

Pandian P K, Emmanuel O, Ruscoe J P, et al. 2010. An overview of recent technologies on wave and current measurement in coastal and marine applications. Journal of Oceanography and Marine Science, 1(1): 1-10

Peirson W L, Banner M L. 2003. Aqueous surface layer flows induced by microscale breaking wind waves. Journal of Fluid Mechanics, 479: 1-38

Romeiser R, Suchand S, Runge H, et al. 2010. First analysis of TerraSAR$\mathrm{X}$ along-track InSAR-derived current fields. IEEE Transactions on Geoscience and Remote Sensing, 48(2): 820-829

Romeiser R, Thompson D R. 2000. Numerical study on the along-track interferometric radar imaging mechanism of oceanic surface currents. IEEE Transactions on Geoscience and Remote Sensing, 38(1): 446-458

Rouault M J, Mouche A, Collard F, et al. 2010. Mapping the Agulhas Current from space: an assessment of ASAR surface current velocities. Journal of Geophysical Research, 115: C10026

Stoffelen A, Anderson D. 1997. Scatterometer data interpretation: estimation and validation of the transfer function CMOD4. Journa of Geophysical Research, 102(C3): 5767-5780

Thompson D R, Jensen J R. 1993. Synthetic-aperture radar interferometry applied to ship-generated internal waves in the 1989 Loch Linnhe experiment. Journal of Geophysical Research Oceans, 98(C6): 10259-10269

Vachon PW, Dobson FW. 1996. Validation of wind vector retrieval from ERS-1 SAR images over the ocean. The Global Atmosphere and Ocean System, 5(2): 177-187

Wu Hui, Zhu Jianrong. 2010. Advection scheme with 3rd high-order spatial interpolation at the middle temporal level and its application to salt water intrusion in the Changiiang Estuary. Ocean Modelling, 33: 33-51

Yang Z, Wang H, Saito Y, et al. 2006. Dam impacts on the Changjiang (Yangtze) River sediment discharge to the sea: the past 55 years and after the Three Gorges Dam. Water Resource Research, 42: W04407 